\title{
Grisel Syndrome: A Rare Complication of Pediatric Adenotonsillectomy
}

\author{
Enrico Maria Amadei* and Claudio Cola \\ Department of Otorhinolaryngology and Audiology, Infermi Hospital, Rimini, Italy
}

*Corresponding author: Enrico Maria Amadei, Department of Otorhinolaryngology and Audiology, Infermi Hospital, Italy.

\author{
Received Date: April 13, 2019 \\ Published Date: April 18, 2019
}

\begin{abstract}
We present our casuistry of pediatric adenotonsillectomies carried out during the period 2009-2018. We report our experience about a rare and often not quickly recognized complication the Grisel syndrome. This consists of a non-traumatic atlantoaxial subluxation with inflammation in adjacent soft tissues. We performed 1231 paediatric adenotonsillectomies and we found 4 cases of Grisel syndrome. We discuss the diagnosis and treatment of this complication.
\end{abstract}

Keywords: Grisel Syndrome; Torcicollis; Pediatric adenotonsillectomy; Complications

\section{Introduction}

We report our casuistry of 1231 pediatric adenotonsillectomies. All were performed at our Hospital during the period 2009-2018 by the same surgeon. In adult patients adenoidectomy is rarely indicated. The main indication for tonsillectomy is given by recurring inflammations/infections. From international guidelines the indication is given by 5 or more episodes of tonsillitis per year, for at least 2 consecutive years [1]. In pediatric patients, on the other hand, the main indication for adenoidectomy is given by nasal breathing difficulty and by recurrent acute or chronic otitis. The indication for tonsillectomy is essentially represented by obstruction of the upper airways caused by hypertrophic tonsils, with consequent night snoring and sleep apnea. Adenotonsillectomy is a routine surgery for an otolaryngologist, but never trivial. In fact, there are many transient problems after surgery: these are mainly represented by the difficulty in swallowing, halitosis, fever, earache, post-tonsillectomy hemorrhage. This last complication has an incidence in our casuistry of $3 \%$ per year, as in the international case studies. In 4 cases we found a persistent torcicollis.

Initially we thought that this torticollis was a consequence of a misunderstood cervical trauma during surgery or following an agitated awakening from anesthesia. In short, we understood that this persistent torcicollis is named "Grisel syndrome". An inflammation of the upper airways, following surgery or infection, it causes a muscular stiffening, up to the atlantoaxial subluxation. This typically occurs in the pediatric population, because the cervical ligaments, and in particular the transverse and alar ligaments of the atlanto-axial joint, are more lax than those of adults.

Some Authors hypothesized that the anatomical explanation of Grisel syndrome lies in the connection between retropharyngeal veins and the periodontoidal venous plexus. This connection could favor the rise of inflammatory material from the pharyngeal region to the first cervical vertebrae. This would generate a ligamentous laxity of already fragile structures for the young age of children and a reflex muscle contraction [2].

\section{Materials and Method}

This case history concerns 1231 pediatric adenotonsillectomies: all these were carried out over a period of 10 years (20092018 ) by the same surgeon. The age of the patients varies from 2 to 14 years, with a peak between 4 and 8 years, without sex prevalence (54\% female- $46 \%$ male). All patients underwent a tonsillectomy or an adenoidectomy or more frequently a combined adenotonsillectomy. The surgery was always performed with cold instruments, coagulating with bipolar electric forceps. All surgeries were performed at the same Hospital.

We found 4 cases of Grisel syndrome: 3 young patients (of ages 4,6 and 7 respectively) were treated with only nonsurgical management, consisting of close reduction and immobilization. An 11-year-old patient developed a persistent torticollis, which required neurosurgical reduction in sedation (Figure 1-3). However, 
a rapid manipulation of the unconscious patient was sufficient to realign the atlantoaxial joint. After this procedure the young patient had to wear a rigid collar for 2 months, with a good final success.

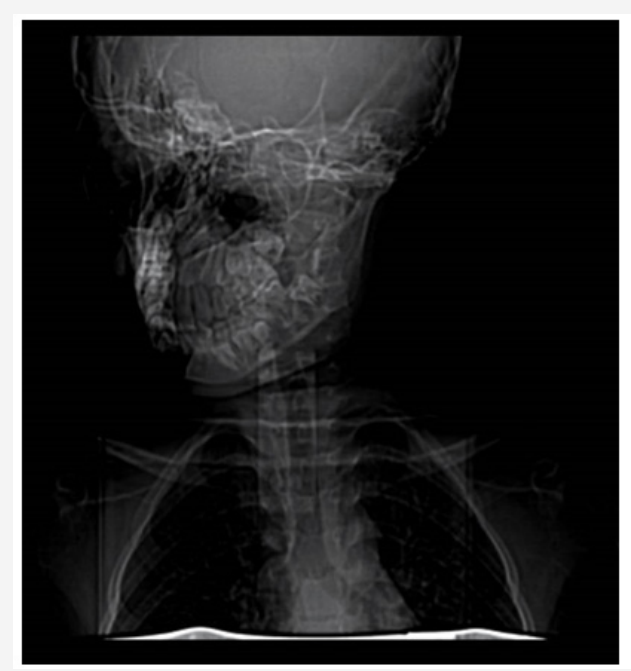

Figure 1: X-ray Pre-CT of an 11-year-old child with Grisel syndrome: typically, torticollis is characterized by tilting of the head to one side and rotation of the head toward the opposite side.

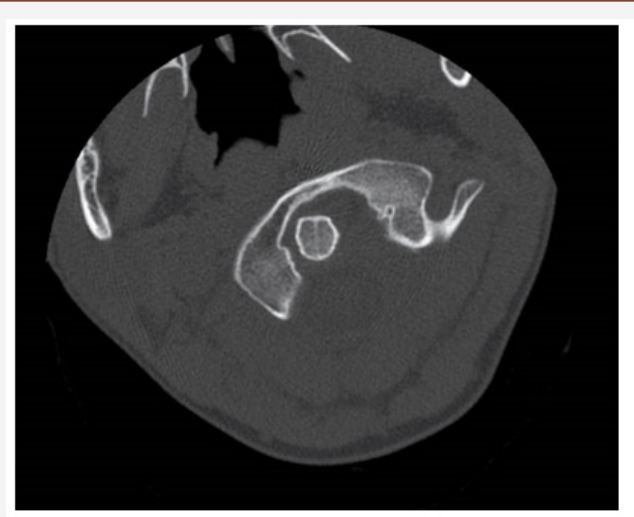

Figure 2: CT scan of an 11-year-old child with Grisel syndrome, axial view: subluxation of $\mathrm{C} 1$ on $\mathrm{C} 2$.

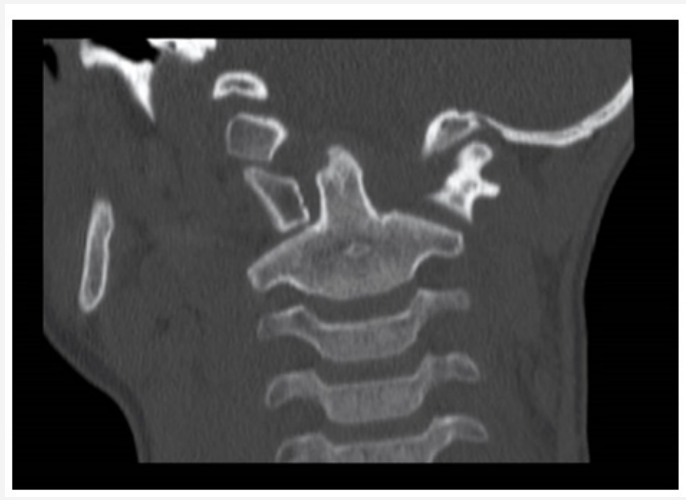

Figure 3: CT scan of an 11-year-old child with Grisel syndrome, lateral view: subluxation of atlanto-axial joint.

\section{Discussion}

Grisel was the French otorhinolaringologist who first described 2 cases of torcicollis consequent to pharyngitis, in French in 1930
[3] and in English in 1951 [4]. From him derives the name of this rare, often not quickly recognized syndrome. A history of ENT surgery or infection, torticollis, and specific radiologic features must be present to define the diagnosis [3]. Some Authors consider ENT surgery (mastoidectomy, adenotonsillectomy), Others airway infections (pharyngitis, otitis, mastoiditis), to be the most frequent cause of Grisel Syndrome [5]. In our experience we never found a Grisel syndrome following an airway infection. All our 4 cases of Grisel syndrome occurred after an adenotosillectomy. Grisel syndrome has an incidence of 3.25/1000 in our casuistry. This is a very interesting data, as in Literature we find many case reports or papers about Grisel syndrome, without indicating its true incidence.

First of all, a cervical pain appears and it generates an initially reversible torticollis. After a few weeks, a fixed atlantoaxial subluxation is established.

Cervical restraint is the best way to prevent the stabilization of subluxation: early cervical immobilization, for at least three months [5], combined with the use of analgesics allows the torticollis to be resolved. If action is taken too late, neurosurgical reduction and realignment is necessary: sometimes arthrodesis of the first and second cervical vertebrae is indicated [6]. The reduction of a stabilized torticollis must be particularly delicate, to avoid the risk of injury compression of the spinal cord by the odontoid process [4].

About radiology, a CT should be performed. CT is important to evaluate the degree of atlantoaxial displacement, and the Fielding and Hawkins grading system is used to do this [7]. In reality we did a CT scan only for the 11-year-old child, because unfortunately his torticollis was not reversible with a simple manipulation when awake. We have checked the child twice in 40 days after surgery: the first one on the seventh day post-surgery and the second one after 40 days. Unfortunately, the torcicollis was established around the eighth-tenth day after surgery, and neither parents nor pediatrician were alarmed by the young patient's stiff neck, because they thought it was a simple transient analgesic position. Indeed, the patient reported no pain with his bent head at rest, but the torcicollis was painful when he tried to straighten his head.

\section{Conclusion}

In conclusion we still do not know why the atlantoaxial subluxation occurs we have always used the same procedure on all patients, by the same surgeon, with the same positioning of the patient during the surgical operation. All patients were in good general condition at the time of surgery. This was always performed in election, and never as an emergency on a febrile patient or on a child with bronchitis. Certainly, some patients are more anatomically predisposed than others to develop Grisel syndrome.

We believe it is essential that every otolaryngologist knows Grisel syndrome very well. It is rare, because in our series the overall incidence is $3.25 / 1000$ during a period of 10 years, but it is severe. We have to think that it is already difficult for parents to decide to have their child face a surgery that has a complete 3-weeks healing prognosis. Then finding their child a few days after adenotonsillectomy with an irreversible torcicollis, it can be a very important shock for parents. 
It is important for us to monitor children who have undergone adenotonsillectomy 7 days after discharge and then again 30 days after surgery. It is essential to create a good relationship of trust with the parents of the operated children and with their pediatricians. So that in case of doubt, timely and effective countermeasures can be adopted. This in order to avoid further surgery and general anesthesia, to repair unpleasant complications, such as the atlantoaxial subluxation.

\section{Conflict of Interest}

No Conflict of Interest.

\section{Acknowledgement}

None.

\section{References}

1. Ron B Mitchell, Sanford M Archer, Stacey L Ishman, Rosenfeld RM, Coles S, et al. (2019) Clinical Practice Guideline: Tonsillectomy in Children (Update). Otolaryngology-Head and Neck Surgery 160(1S): S1-S42.
2. Nukhet Aladag Ciftdemir, Tuba Eren, Mert Ciftdemir (2018) A Rare Cause of Torticollis: Grisel Syndrome. Journal of Tropical Pediatrics 64(3): 245-248.

3. Ortega Evangelio G, Alcon JJ, Alvarez pitti J, Sebastia V, Juncos M, et al. (2011) Eponym: Grisel syndrome. Eur J Pediatr 170(8): 965-968.

4. Ajit Gupta, Jay Prakash, Pawan Kumar, Niraj Narain Singh (2017) Anesthetic issues and Difficult Airway Management in a Case of Grisel's Syndrome. Anesth Essays Res 11(4): 1094-1096.

5. Pasquale Anania, Piero Pavone, Mattia Pacetti, Monica Truffelli, Marco Pavanello, et al. (2019) Grisel Syndrome in Pediatric Age: A Single-Center Italian Experience and Review of the Literature. Worl Neurosurgery 125: $374-382$.

6. Spennato P, Nicosia G, Rapanà A, Cicala D, Donnianni T, et al. (2015) Grisel Syndrome Following Adenoidectomy: Surgical Management in a Case with Delayed Diagnosis. World Neurosurg 84(5): 1494.e7-12.

7. Fielding JW, Hawkins RJ (1977) Atlanto-axial rotatory fixation (fixed rotatory subluxation of the atlantoaxial joint). J Bone Jt Surg 59(1): 3744. 